\title{
Diagnostic Dilemma with a Case of Primary Bronchopulmonary Actinomycosis Causing Unilateral Destroyed Lung: A Case Report and Literature Review
}

\author{
Md. Abir Tazim Chowdhury ${ }^{1}$, Sohail Ahmed', Md. Zulfiqur Haider ${ }^{1}$, S. M. A. Zulker Nine1, \\ Md. Kamrul Hasan', Md. Estiak Ullah Sahid1, Musfaqus Shalehin1, \\ Md. Abdullah Al-Mahammud Kabir², Niaz Ahmed2, Munama Magdum³
}

${ }^{1}$ Department of Cardiothoracic and Vascular Surgery, Evercare Hospital, Dhaka, Bangladesh

${ }^{2}$ Department of Cardiothoracic Anesthesia, Evercare Hospital, Dhaka, Bangladesh

${ }^{3}$ Department of Community Medicine, Shaheed Monsur Ali Medical College, Dhaka, Bangladesh

Email: * chowdhuryabir0@gmail.com

How to cite this paper: Chowdhury, Md.A.T., Ahmed, S., Haider, Md.Z., Zulker Nine, S.M.A., Hasan, Md.K., Sahid, Md.E.U., Shalehin, M., Kabir, Md.A.A.-M., Ahmed, N. and Magdum, M. (2022) Diagnostic Dilemma with a Case of Primary Bronchopulmonary Actinomycosis Causing Unilateral Destroyed Lung: A Case Report and Literature Review. Open Journal of Thoracic Surgery, 12, 1-10.

https://doi.org/10.4236/ojts.2022.121001

Received: December 14, 2021

Accepted: January 26, 2022

Published: January 29, 2022

Copyright $\odot 2022$ by author(s) and Scientific Research Publishing Inc. This work is licensed under the Creative Commons Attribution International License (CC BY 4.0).

http://creativecommons.org/licenses/by/4.0/

\begin{abstract}
Among chronic pulmonary infections, pulmonary actinomycosis is a rare one, caused by a gram-positive microaerophilic bacterium called Actinomyces. Aside from cervicofacial or abdominopelvic actinomycosis, pulmonary involvement is rare, and sometimes this type of infection results in a misdiagnosis of pulmonary tuberculosis or carcinoma. Clinical presentation of pulmonary actinomycosis could be acute or subacute, with lobar involvement. However, the disease is most commonly diagnosed at the chronic phase, with patients presenting mild fever, weight loss, and occasional hemoptysis. Here, we described the case of a 30-year-old male patient, presented with a history of respiratory complaints for more than three years and was diagnosed clinically and radiologically as a case of a destroyed left lung. Later on, post-operative tissue diagnosis was confirmed-pulmonary actinomycosis.
\end{abstract}

\section{Keywords}

Pulmonary Actinomycosis, Destroyed Lung, Diagnostic Dilemma

\section{Introduction}

Actinomycosis is a chronic granulomatous slow progressive inflammatory disease caused by filamentous gram-positive anaerobic or microaerophilic bacteria of the family Actinomycetaceae (genus Actinomyces). These bacteria mainly be- 
long to the human commensal flora of the oropharynx, gastrointestinal tract, and urogenital tract. However, various anatomical sites can be affected (e.g., the face, bone and joint, respiratory tract, genitourinary tract, digestive tract, central nervous system, skin, and soft tissue structures) by Actinomyces infection; with multitudes of different clinical features [1]. It is assumed that pulmonary actinomycosis infection is caused primarily by the inhalation or aspiration of oropharyngeal or upper gastrointestinal substances. This pulmonary form of the disease accounts for $15 \%$ of all actinomycosis cases [2]. The nature of the spread of actinomycosis infection is progressive, and its involvement in any site frequently mimics malignancy, tuberculosis, or nocardiosis. Hence, an initial diagnosis of actinomycosis is, therefore, both tough as well as challenging [1] [3]. Here, we reported a postoperative histopathologically diagnosed case of pulmonary actinomycosis, which had presented with clinical and radiological features suggestive of the destroyed left lung.

\section{Case Presentation}

A 30-year-old male patient with a destroyed left lung was admitted to our hospital for evaluation. He had no notable medical history besides a smoking history of 10 pack-years. He was presented with a chronic productive cough with mucoid expectoration and occasional hemoptysis for more than three years. And for the last few weeks, he has been facing difficulty breathing. Moreover, he complained of a gradual weight loss of 10 kilograms in the previous 12 months. In his initial course of the disease, he took anti-tuberculosis treatment empirically for six months advised from a rural health center. But as symptoms were not improving, he visited several hospitals for evaluation. He was evaluated with different diagnostic tools, i.e., computed tomography (CT) chest (Figure 1), bronchoscopy (Figure 2), and spirometric pulmonary function test (Figure 3). Where, CT scan of the chest findings suggestive of bronchiectatic left lung, Bronchoscopy reported with thick purulent bronchial secretion suggestive of infective lung disease and Spirometric pulmonary function revealed restrictive lung disease. Bronchoscopy-guided bronchial wash was examined with Gram, Acid-fast bacillus (AFB), and fungal stain to identify any causative agents. The gram stain revealed plenty of pus cells and gram-positive cocci in pairs and scattered patterns. However, AFB and fungal staining revealed nothing. The physician did a gene X-Pert MTB (Mycobacterium tuberculosis) test of sputum, showing no positive MTB complex results. A blood hemogram revealed-Hemoglobin-10.4 $\mathrm{gm} / \mathrm{dl}$ with an erythrocyte sedimentation rate (ESR) $91 \mathrm{~mm} / \mathrm{in} 1^{\text {st }}$ hour. With the above history and diagnostic parameters, he was treated medically in several different centers as a post-tuberculosis squeal case of bronchiectasis. As the patient's symptoms become worsened, he came to our hospital for better management. On physical examination, the patient had: mildly anemic, afebrile, weighing 48 kilograms, poor oral hygiene, without any accessible palpable lymph node, blood pressure $90 / 60 \mathrm{~mm}$ of $\mathrm{Hg}$, heart rate 130 /minute, respiratory rate 18 
breath/minute, and oxygen saturation $91 \%$ in room air. On chest examination, the patient had: restricted chest expansion, tracheal deviation, dull percussion notes, and diminished breath sounds all over the left side of the chest. However, the right side was revealed reasonably healthy with features of the hyper-inflated lung. A recent chest radiograph after admission of the patient showed a significant loss of left lung volume, with a slight deviation of the trachea to the left,

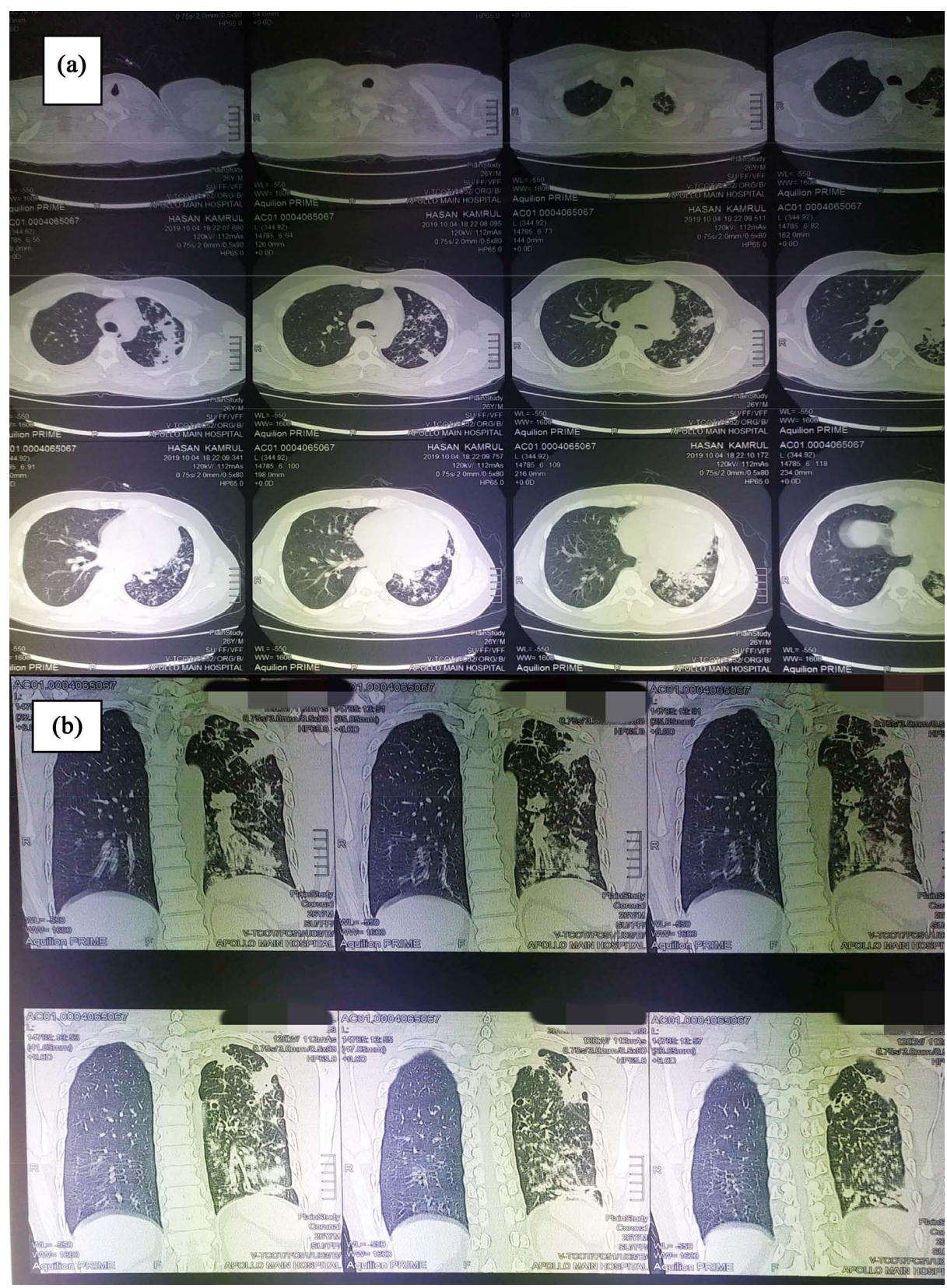

Figure 1. Computed tomography (CT) of chest. Showing multiple nodular lesions of variable sizes in left upper and lower lobes, many of them showing cavitation and there is also associated bronchiectasis with peribronchial thickening and nodular septal thickening. Pleural thickening and fibrotic strands with calcification in left upper lobe. Small nodular lesions with fibrotic bands also noted in right upper lobe and middle lobes. a) Axial slice, b) Coronal slice of CT chest, respectively. 


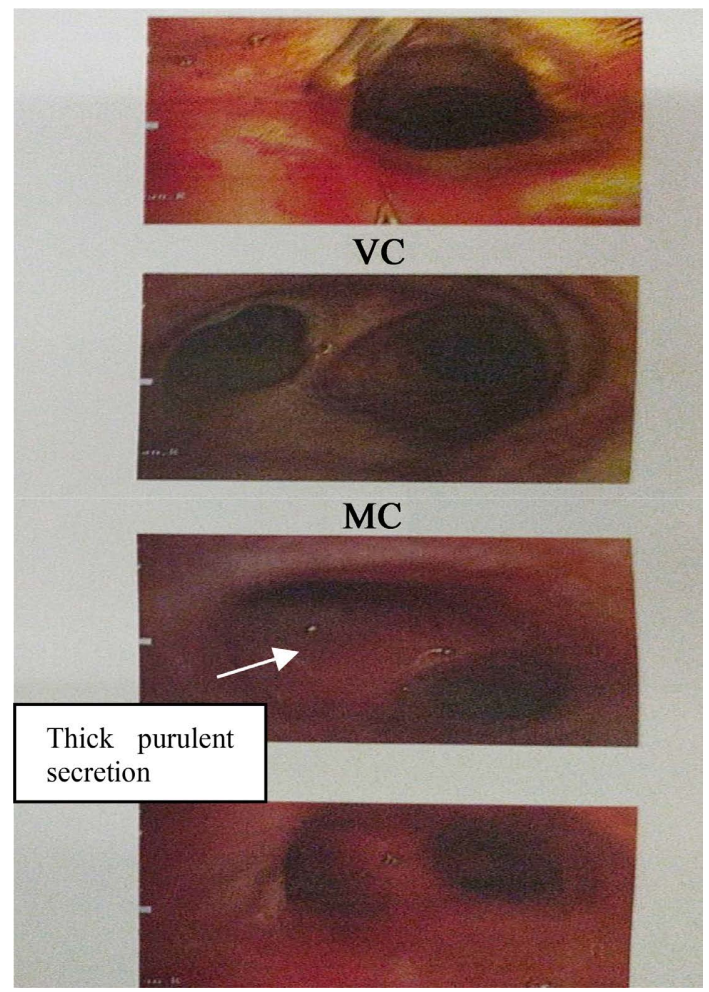

Figure 2. Bronchoscopy report. Showing normal vocal cords (VC), main carina (MC), but thick purulent secretions in left lung.

\begin{tabular}{lrrrrrrr} 
FVG & [L] & $\begin{array}{r}\text { Pred } \\
4.58\end{array}$ & $\begin{array}{r}\text { Pre } \\
1.75\end{array}$ & $\begin{array}{r}\% \text { (Obs/F } \\
38.1\end{array}$ & $\begin{array}{r}\text { Post } \\
1.77\end{array}$ & $\begin{array}{r}\%(\text { Obs/F } \\
38.7\end{array}$ & $\begin{array}{r}\text { D\%(2/1) } \\
1.5\end{array}$ \\
\hline FEV 1 & [L] & 3.91 & 1.34 & 34.4 & 1.46 & 37.4 & 8.8 \\
\hline FEV 1 \% FVC & [\%] & & 77.03 & & 82.54 & & 7.1 \\
\hline MEF 75 & [Us] & 7.87 & 3.17 & 40.4 & 3.12 & 39.6 & -1.8 \\
\hline MEF 50 & [U/s] & 5.14 & 1.43 & 27.8 & 1.75 & 34.1 & 22.5 \\
\hline MEF 25 & [U/s] & 2.32 & 0.43 & 18.4 & 0.57 & 24.7 & 34.3 \\
\hline PEF & [U/s] & 9.24 & 3.34 & 36.1 & 3.35 & 36.3 & 0.5 \\
\hline PIF & [Us] & & 3.12 & & 2.89 & & -7.6 \\
\hline FET & [s] & & 5.36 & & 5.43 & & 1.2
\end{tabular}
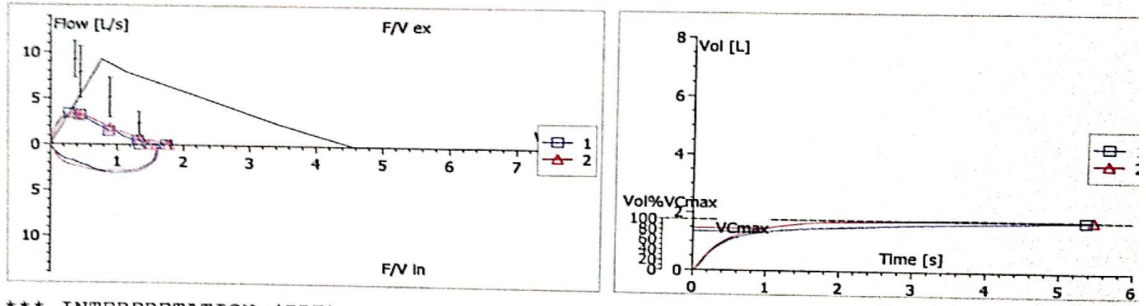

*** INTERPRETATION (PRE)

Severe expiratory flow limitation.

Further to highly distinctive restrictive shape of curve.

***

Moderate expinatory flow

Moderate

Further lung function measurment rective shape of curve.

Figure 3. Spirometric pulmonary function test report. It revealed-in pre broncho-dilation state, severe expiratory flow limitation and in post dilatation state moderate expiratory flow limitation, and in both this state there was moderate to highly distinctive restrictive shape of curve. 
and there was hyperinflated contralateral lung (Figure 4). With above mentioned clinical and diagnostic evidence, we took respiratory medicine consultation. Finally, surgical removal of the left lung was planned by our surgical team. The patient was thoroughly counseled regarding the surgery and then prepared for a standard thoracotomy.

After proper aseptic precaution and under general anesthesia with doublelumen endotracheal intubation, before positioning the patient for surgery, we performed a fiber optic bronchial examination, pathologies were noted, and our surgical team also confirmed the tube's position. Later on, the patient positioning was done for the left postero-lateral thoracotomy. Into Left hemithorax was entered through $5^{\text {th }}$ inter-costal space, there was excessive adhesion of the lung with surrounding structures-fibrotic-firm nodular consistency throughout the lung parenchyma. Lung hilar arrangements were disease-free. With the above findings, the per-operative decision was made for left pneumonectomy. With ensuring a disease-free healthy bronchial stump, pneumonectomy was performed, and tissue specimen was sent for histopathological examination. The chest was closed in layers following adequate hemostasis with a single intercostal drain in situ. The patient was shifted to the cardiothoracic intensive care unit with a stable hemodynamic state. After 24 hours of monitoring, the patient was moved to a surgical ward. On the $3^{\text {rd }}$ postoperative day, the histopathological report of the resected lung tissue came. It showed cotton ball basophilic appearance with neutrophilic infiltrates in the background and filamentous bacterial with dark sulfur granules, in Hematoxylin \& eosin (H\&E) and Groccot-Gomori methamine silver stain, respectively. These microscopic findings of above mentioned histopathological slides confirmed bronchopulmonary actinomycosis (Figure 5

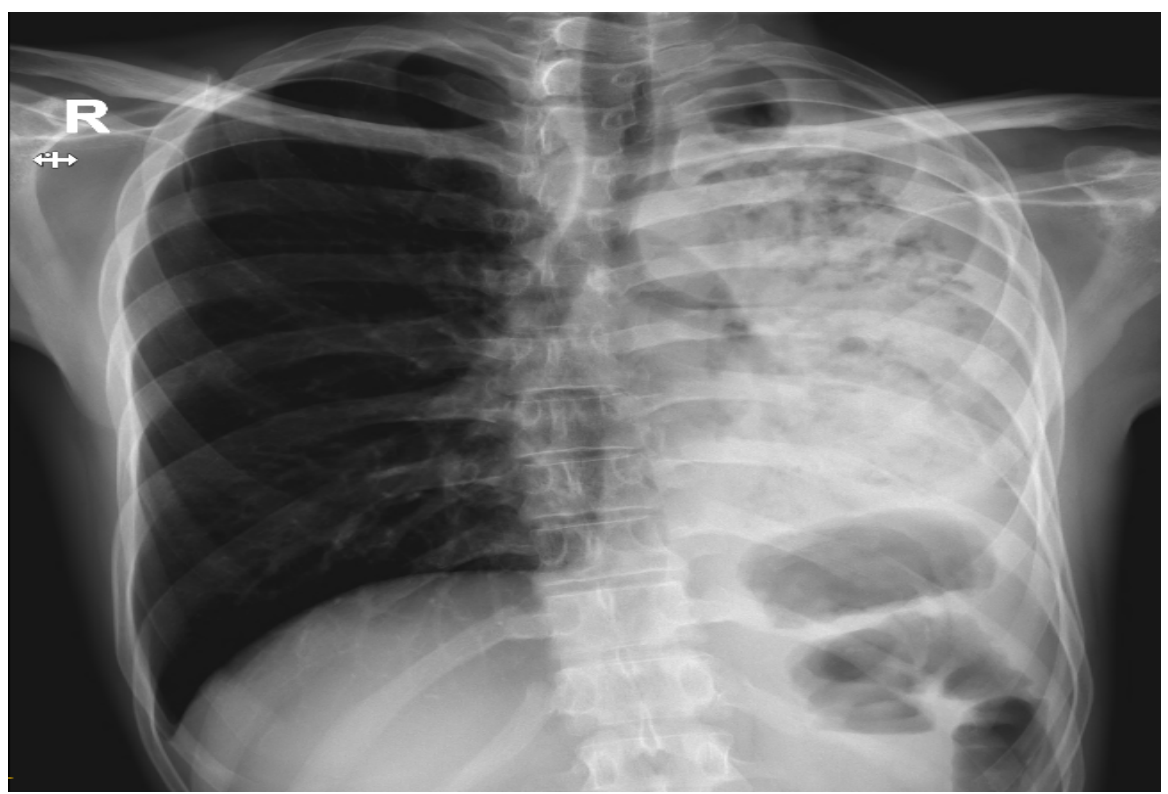

Figure 4. Preoperative posterior-anterior chest radiograph showing marked loss of left lung volume, with slight deviation of trachea to left and there was hyperinflated contralateral lung. 
\& Figure 6). On the $7^{\text {th }}$ postoperative day, a chest radiograph (Figure 7) was taken and found increased fluid level and decreasing gas shadow in the left hemithorax, and then the chest drain tube was removed by our team uneventfully.

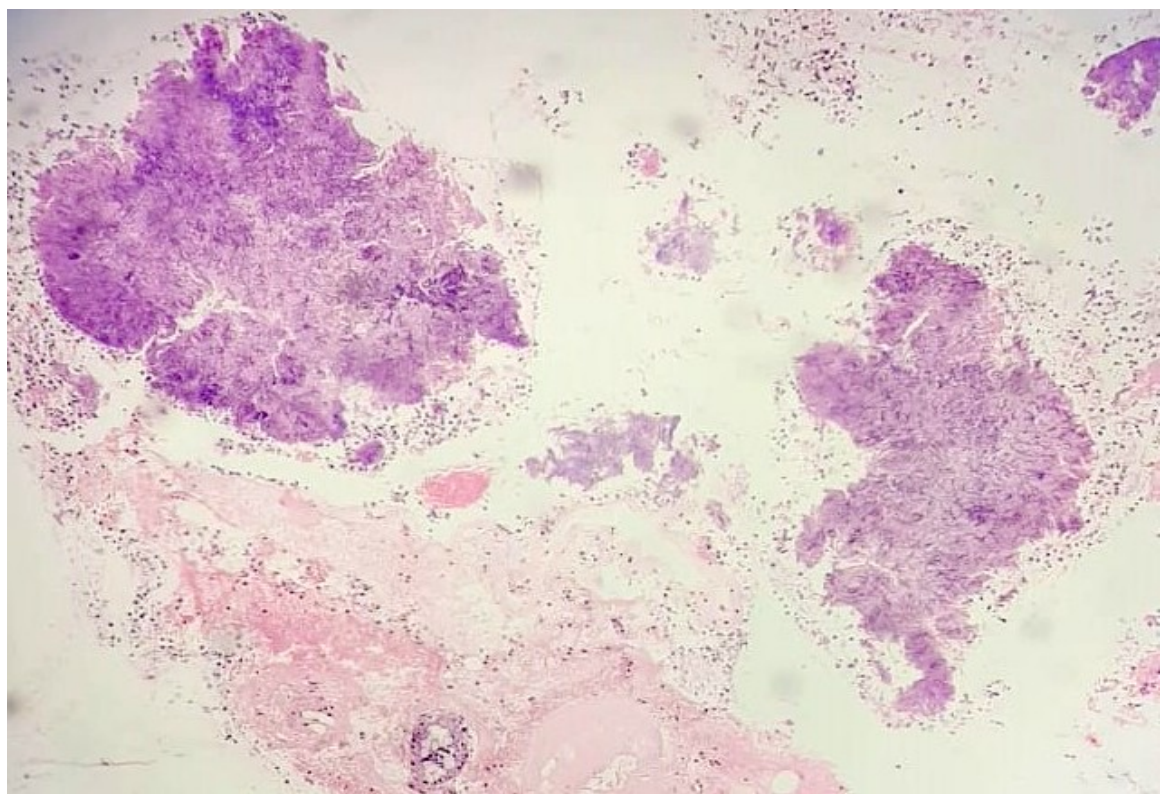

Figure 5. Hematoxylin \& eosin (H\&E) section showing cotton ball basophilic appearance with neutrophilic infiltrates in the background $(20 \times)$.

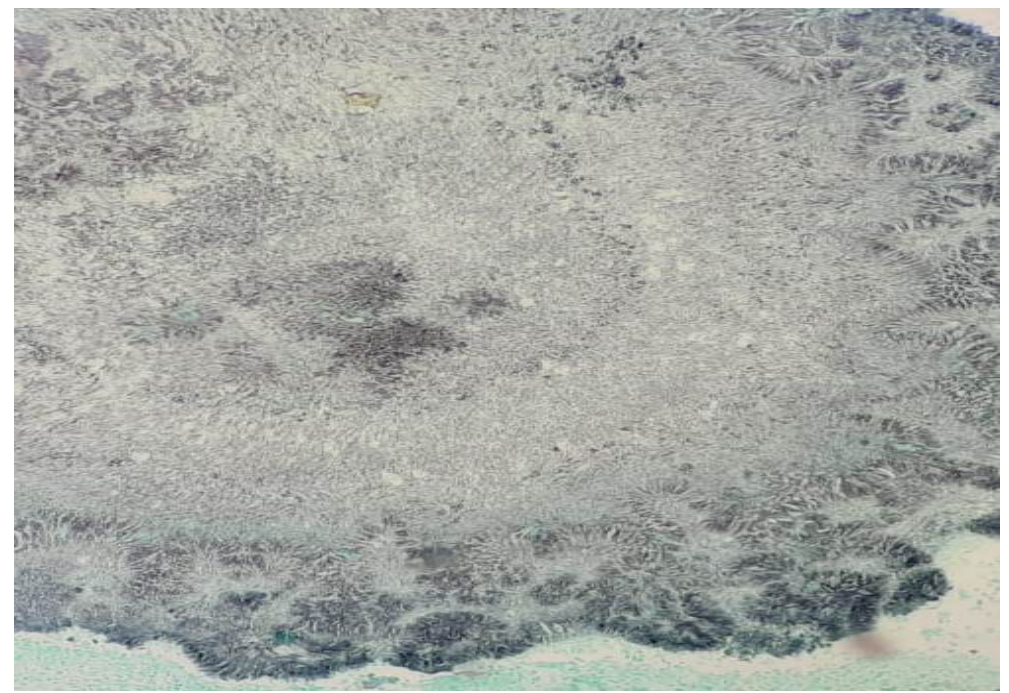

Figure 6. Groccot-Gomori methamine silver stain showing filamentous bacterial with dark sulphur granules $(40 \times)$

The patient was discharged home uneventfully with continuing antibiotic Tablets. AMOXICILLIN 500 milligram + CLAVULANIC ACID 125 milligram thrice daily for two weeks, and Tablet. PHENOXYMETHYL PENICILLIN 250 mg twice daily for 12 weeks. The patient was followed up initially at a 1-month interval than six months. At one year following surgery, the patient reported to 
be free of any respiratory complaints and gained weight. Chest radiograph demonstrated regular post-pneumonectomy changes (Figure 8).

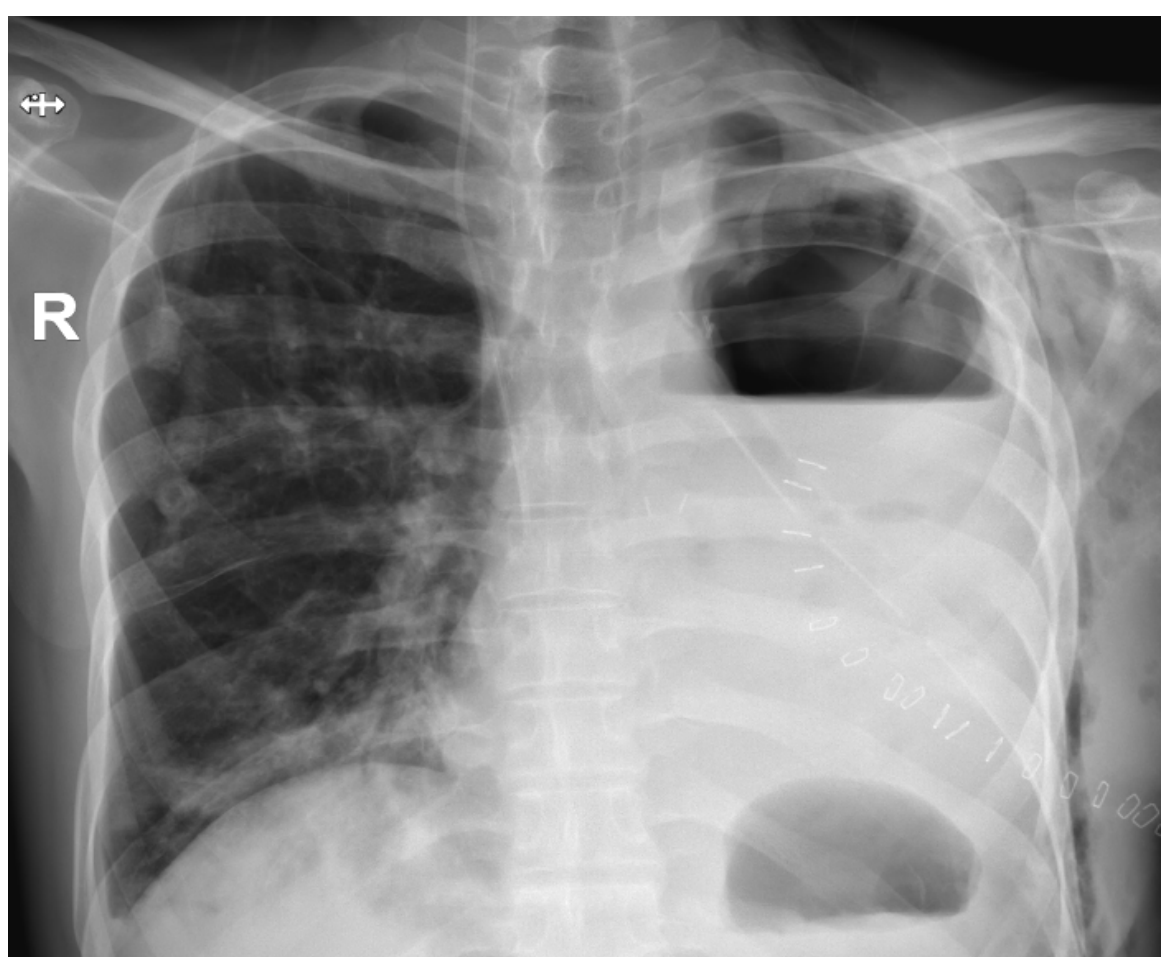

Figure 7. Post-pneumonectomy chest X-ray on $7^{\text {th }}$ postoperative day. Showing increasing fluid level and decreasing gas within the left hemithorax.

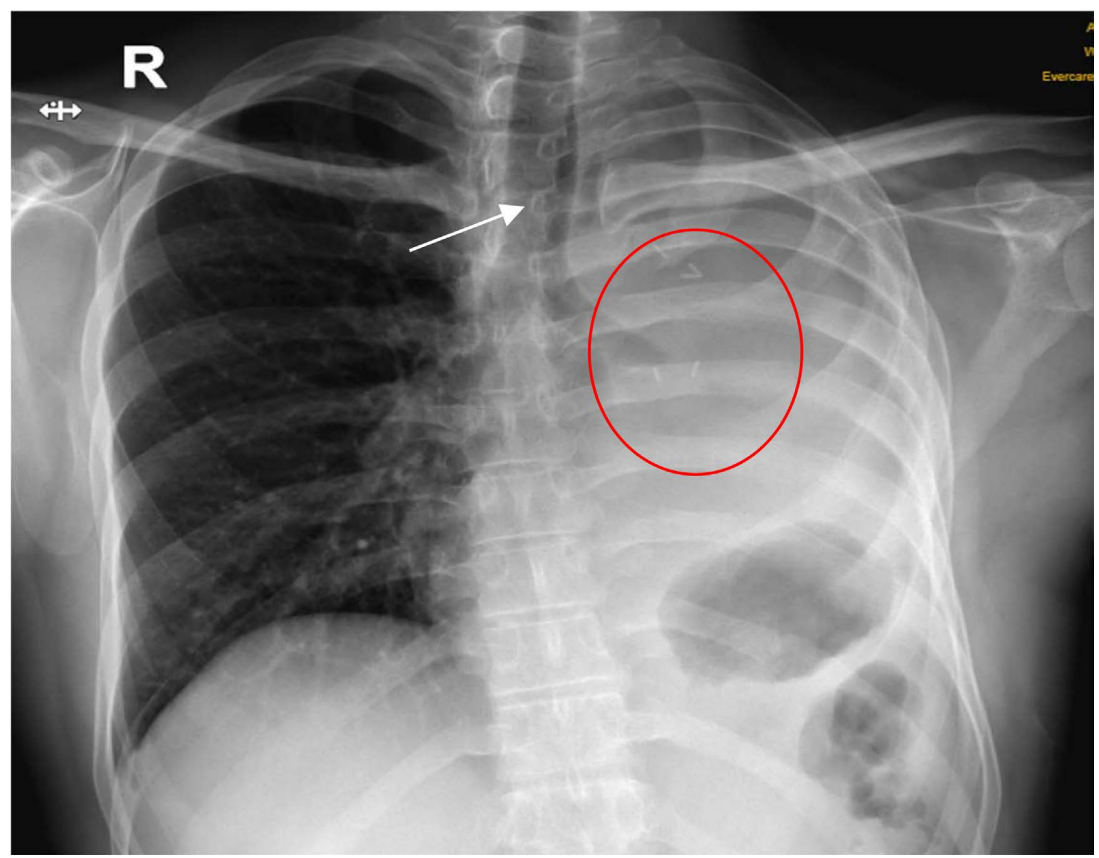

Figure 8. Post-pneumonectomy Chest X-ray at the end of $1^{\text {st }}$ year after surgery. Showing complete opacification of the left hemithorax with shifting of the heart and mediastinal structures, such as the trachea (white arrow) towards the left indication volume loss. Multiple surgical clips are seen adjacent to the stump of the left main bronchus (red circle). 


\section{Discussion}

Actinomycosis of the respiratory tract comprises pulmonary, bronchial, and laryngeal actinomycosis. In literature, pulmonary actinomycosis is the third most frequent site of infection after cervicofacial and abdominoperineal involvement. Its peak age incidence is reportedly in the $4^{\text {th }}$ and $5^{\text {th }}$ decades and predominantly affects men (Male:Female $=3: 1$ ). Aspiration of oropharyngeal or gastrointestinal contents is one of the primary causes; however, locally spread from the cervicofacial region, and hematogenous spread also can occur. Among the risk factors of pulmonary actinomycosis, the most important risk factors are poor oral hygiene, preexisting dental illness, and alcohol consumption. Additionally, chronic pulmonary disease (e.g., chronic obstructive lung disease, bronchiectasis, chronic mycobacterial disease, and aspergilloma) are also considered risk factors of pulmonary actinomycosis. In those cases, damaged pulmonary tissues are provided a favorable anaerobic environment for the growth of actinomycetes [3] [4] [5] [6]. Although smokers or diabetic patients have been described as the most vulnerable for pulmonary actinomycosis. However, recent data showed that nearly half of actinomycosis patients are not suffering from any comorbidity. It reflects that thoracic actinomycosis does not occur only in comorbid patients. About $50 \%$ of the patients were documented taking antibiotic treatment before hospitalization and might be suspicious of the presence of rare organisms, such as actinomycetes [6]. Most of the symptoms of bronchopulmonary actinomycosis are nonspecific. But among them, cough, productive sputum, hemoptysis, chest pain, weight loss, fever, and dyspnea are commonest. These symptoms are usually shown after the lapse of several months, and these are also mimicking a variety of other pulmonary pathologies, viz. tuberculosis, malignancy, pneumonia, and pulmonary abscesses, which are reasons behind concluding the diagnosis more difficult [7]. The most common conventional diagnostic tools to evaluate such symptoms are radiological imaging, bacteriological studies, image-guided or direct tissue biopsies, and histopathological examination. In addition, newly developed molecular methods, such as PCR with 16S rRNA gene sequencing and mass spectrometry, seem to provide rapid and precise microbiological confirmation of the disease [8] [9]. However, these newly developed techniques are still not popular because they are not widely available to use in routine clinical practice, and their insufficient clinical data on their sensitivity and specificity for clinical decision-making. The commonest radiological finding is a solidification of the lung parenchyma, and the others are-mediastinal and hilar lymphadenopathy, atelectasis, ground-glass opacity, and pleural angle obliteration [8]. Even though microbiological culture studies to identify and confirm the causative organism should carry out, it has been achieved in only a minority of cases. And this is notably because of pretreatment of the symptomatic cases with empirical antimicrobial, overgrowth of associated bacteria, and the fastidious nature of actinomycetes. However, the diagnosis of pulmonary actinomycosis is substantially based on histopathological examination of pulmonary tis- 
sue biopsies obtained by image-guided transthoracic needle biopsy, bronchoscopic techniques, or even surgical resection. The typical histopathological features of pulmonary actinomycosis observed on staining with hematoxylin-eosin is-sulfur granules are colonies of organisms that appear as round or oval basophilic masses with radiating eosinophilic terminal clubs [1] [2] [8]. Therefore, the recommended diagnostic algorithm either starts with radiological imaging-guided percutaneous procedures or bronchoscopic techniques before considering any surgical approach. Surgery may lead to considerable morbidity, discomfort, costs, and diagnostic delay [8]. Medical management with appropriate antibiotics is generally curative, and for that, timely management of pulmonary actinomycosis has a good prognosis. Failure of medical treatment can result in patients, those who are not strictly consuming the prescribed antibiotics. A recently published study reported that no response of an antibiotic at one month is the only independent factor associated with a poor treatment outcome [8] [10]. Treatment of choice of antibiotics and dosage formulation is-Intravenous penicillin at a dose of 18 - 24 million units daily for $2-6$ weeks, followed by oral penicillin or amoxicillin for 6 - 12 months. Surgical management should primarily be performed on patients presented with specific complications (e.g., massive hemoptysis, pleural empyema, damaging lung parenchyma, etc.). Besides the above measures, the most important step is reducing existing risk factors. Such as restoration of a poor dental status, management of aspiration syndromes, or therapy of impaired immune function, and these should be included in the overall management scheme and need to ensure continuity throughout the treatment process [8].

At the endpoint of discussion, in this reported case, we noted the following key points that made the diagnostic dilemma; these were:

1) initial diagnosis, i.e., pulmonary tuberculosis was not conclusive, and it was not evidence-based;

2) first microbiological specimen of bronchial wash of the patient revealed infective gram-positive cocci. However, there was no evidence of bacteriological identification. The patient was treated with antibiotics, but he was not in follow-up with prescribing physician;

3) throughout his illness, there was no evidence of any tissue diagnosis;

4) At the presentation, symptoms were worse and pulmonary damage was extensive.

So we planned to remove the diseased lung surgically and diagnose the case histopathologically without prior image-guided tissue diagnosis.

\section{Conclusion}

In conclusion, pulmonary actinomycosis is one of the rare and slowly progressing bacterial lung disease, and its nature of progression sometimes makes poor compliance to prescribed treatment and follow-up. Due to its mimicking nature with other chronic pulmonary destructive diseases, viz. pulmonary tuberculosis, 
pulmonary malignancy, or bronchiectasis, respiratory physicians should be vigilant during diagnosis by performing all the appropriate diagnostic tools (e.g., CT-guided transthoracic biopsy and bronchoscopic techniques) to exclude it. If there is any confusion, don't hesitate to do more invasive methods (e.g., surgery). It's far usually a curable pathology provided that it is early identified.

\section{Conflicts of Interest}

The authors declare no conflicts of interest regarding the publication of this paper.

\section{References}

[1] Wong, V.K., Turmezei, T.D. and Weston, V.C. (2011) Actinomycosis. BMJ, 343, Article ID: d6099. https://doi.org/10.1136/bmj.d6099

[2] Mabeza, G.F. and Macfarlane, J. (2003) Pulmonary Actinomycosis. European Respiratory Journal, 21, 545-551. https://doi.org/10.1183/09031936.03.00089103

[3] Valour, F., Sénéchal, A., Dupieux, C., Karsenty, J., Lustig, S., Breton, P., Gleizal, A., Boussel, L., Laurent, F., Braun, E. and Chidiac, C. (2014) Actinomycosis: Etiology, Clinical Features, Diagnosis, Treatment, and Management. Infection and Drug Resistance, 7, 183-197. https://doi.org/10.2147/IDR.S39601

[4] Bates, M. and Cruickshank, G. (1957) Thoracic Actinomycosis. Thorax, 12, 99. https://doi.org/10.1136/thx.12.2.99

[5] Apothéloz, C. and Regamey, C. (1996) Disseminated Infection Due to Actinomyces meyeri: Case Report and Review. Clinical Infectious Diseases, 22, 621-625. https://doi.org/10.1093/clinids/22.4.621

[6] Kalditz, M. and End, A. (2013) Actinomycosis of the Lung and Pleura. Complex Pleuropulmonary Infections: European Respiratory Monograph, 61, 66-80. https://doi.org/10.1183/1025448x.10040912

[7] Han, J.Y., Lee, K.N., Lee, J.K., Kim, Y.H., Choi, S.J., Jeong, Y.J., Roh, M.S. and Choi, P.J. (2013) An Overview of Thoracic Actinomycosis: CT Features. Insights into Imaging, 4, 245-252. https://doi.org/10.1007/s13244-012-0205-9

[8] Katsenos, S., Galinos, I., Styliara, P., Galanopoulou, N. and Psathakis, K. (2015) Primary Bronchopulmonary Actinomycosis Masquerading as Lung Cancer: Apropos of Two Cases and Literature Review. Case Reports in Infectious Diseases, 2015, Article ID: 609637. https://doi.org/10.1155/2015/609637

[9] Ng, L.S.Y., Sim, J.H.C., Eng, L.C., Menon, S. and Tan, T.Y. (2012) Comparison of Phenotypic Methods and Matrix-Assisted Laser Desorption Ionisation Time-ofFlight Mass Spectrometry for the Identification of Aero-Tolerant Actinomyces spp. Isolated from Soft-Tissue Infections. European Journal of Clinical Microbiology \& Infectious Diseases, 31, 1749-1752. https://doi.org/10.1007/s10096-011-1496-3

[10] Park, J.Y., Lee, T., Lee, H., Lim, H.J., Lee, J., Park, J.S., Cho, Y.J., Park, Y.S., Lee, C.H., Lee, S.M. and Yoon, H.I. (2014) Multivariate Analysis of Prognostic Factors in Patients with Pulmonary Actinomycosis. BMC Infectious Diseases, 14, Article No. 10. https://doi.org/10.1186/1471-2334-14-10 